\title{
Policy prescriptions to fix our ailing delivery system
}

\author{
Matt Brow, ${ }^{1}$ Ted Okon, MBA, ${ }^{2}$ Barry Brooks, MD, ${ }^{1,3}$ and Mark Thompson, $\mathrm{MD}^{2,4}$ \\ ${ }^{1}$ The US Oncology Network and McKesson Specialty Health, Washington, DC; ${ }^{2}$ Community Oncology Alliance, Washington, \\ DC; ${ }^{3}$ Texas Oncology, Dallas; ${ }^{4}$ The Mark H. Zangmeister Center, Columbus, Ohio
}

$\mathrm{W}$ e are proud to represent the principal contributors to the world's most effective and successful cancer care delivery system: oncologists and allied medical professionals who care for Americans who are battling cancer in community clinics throughout the United States. The success of these women and men on the frontlines is clear: after nearly 100 years of increasing cancer death rates in the United States, cancer mortality has fallen 20\% from its 1991 peak. Cancer patients from around the world seek care here because Americans enjoy the best cancer survival rates in the world. Yet we still have not realized our potential to eradicate cancer. The American Cancer Society has estimated that 1.6 million Americans were diagnosed with cancer in 2013 and that more than 580,000 will die of the disease during that time. As has been the case for decades, only cardiovascular disease will kill more Americans.

To win this important fight, we need a stable and sustainable cancer care delivery system. That's where Medicare and community-based cancer clinics are so important. Community cancer clinics provide patients with convenient, comprehensive, state-of-theart cancer treatment facilities close to home. And more than $60 \%$ of cancer patients rely on Medicare to pay their medical bills. As the single largest payer of cancer care, Medicare has inordinate influence on the health care delivery system and often guides how private insurers pay for cancer care. As a result, Medicare policies have an impact on cancer care for all Americans, not just those who are covered by Medicare.

\section{A cancer care delivery system in crisis}

During 2013, the national consciousness has been raised about the tremendous strain imperiling our nation's cancer care delivery system. Just 8 years ago, $87 \%$ of cancer care was delivered in cost-effective

Commun Oncol 2013;10:368-370 DOI: 10.12788 / j.cmonc.0080 community oncology clinics. ${ }^{1}$ By 2011 , this percentage had dropped to $67 \%$ as Medicare policies have created an environment in which community cancer clinics are forced to operate at a loss when treating seniors who have cancer. And sadly, the flight from community oncology did not end in 2011. Since early 2012, there has been a $20 \%$ increase in clinic closings and hospital acquisitions, which means that increasingly more patients are facing reduced access to more expensive care. ${ }^{2}$

The data are clear: our world-class community cancer care delivery system is struggling to survive. Since 2008, 1,338 community cancer centers have closed, consolidated, or reported financial problems. Over the past several years, the country has experienced a shift of outpatient cancer care delivery from the physician office to the hospital outpatient department; 288 oncology treatment clinics have closed, and 469 oncology groups have entered into an employment or professional services agreement with a hospital. ${ }^{2}$

When clinics close their doors, access to care is compromised for all cancer patients, but especially for vulnerable seniors in rural areas. The shift to hospital-based care doesn't just reduce access to care for cancer patients, it also increases costs to Medicare, taxpayers, and patients. According to a 2013 report by The Moran Company, outpatient hospital cancer care results in the use of more chemotherapy, more expensive chemotherapy, and a 25\%-47\% higher cost to Medicare and seniors. ${ }^{3} \mathrm{~A}$ 2011 Milliman study found that when care is provided in community cancer clinics, Medicare and seniors pay less overall - the total Medicare spending on chemotherapy patients who receive treatment in the clinic setting is $\$ 6,500$ less per cancer patient annually or about $\$ 623$ million a year. ${ }^{4}$ These differences are even greater for care that is covered by private insurers. ${ }^{5,6}$ Furthermore, there is no clinical justification for the migration of outpatient cancer care to the hospital setting. 


\section{A string of problems..}

\section{Medicare payments for chemotherapy}

In the Medicare Modernization Act of 2003 (MMA), Congress enacted the average sales price (ASP) reimbursement methodology for Part B drugs. Pursuant to the MMA statute, Medicare reimburses community cancer clinics for chemotherapy and related cancer treatment drugs at ASP plus a $6 \%$ services fee to reimburse community cancer clinics for the operational complexity and financial risks associated with purchasing, storing, mixing, administering, and disposing of these highly potent therapies. This $6 \%$ services fee is incredibly important because none of the work that must occur to prepare chemotherapy for administration to a patient is otherwise reimbursed by Medicare. In addition, many states require very specific infrastructure and personnel for the storage and preparation of these drugs, which have cost implications for the cancer clinics.

The current Medicare reimbursement structure for Part $\mathrm{B}$ drugs is not perfect, but it has achieved many of the goals of the policymakers who designed it. It has clearly created a more accurate reimbursement approach than did the previous system and it has attenuated the previous significant growth rate of Part B drug units and spending, thereby creating stability in the costs to Medicare and the patients who rely on it. However, there are technical flaws with the ASP formula that make the Medicare drug reimbursement rates at ASP plus 6\% a theory and not the reality. First of all, the Centers for Medicare and Medicaid Services (CMS) has interpreted the MMA to require manufacturers to include wholesaler prompt-pay discounts in their ASP calculations, even though these discounts are financing terms between manufacturers and wholesalers and are not available to clinics. The inclusion of these discounts artificially reduces ASP by $1 \%-2 \%$. In addition, Medicare reimbursement rates for cancer drugs are based on ASPs that are 2 quarters old, so routine drug price increases are perpetually subsidized by community cancer clinics for 6 months, which results in another 1\%-2\% reduction in the real ASP.

While the prompt-pay discount and 2-quarter lag problems make it difficult for community oncology clinics to break even at ASP plus 6\%, it is quite rare for clinics to be able to collect the entire Medicare allowable rate for cancer drugs. This is principally because of the $20 \%$ coinsurance responsibility of Medicare beneficiaries, often on very expensive therapies. It has been the experience of practices in The US Oncology Network that about 25\% of the beneficiary coinsurance (about $5 \%$ of the Medicare allowable) is uncollectible and ends up as bad debt. While this is meaningful even in the context of cancer care services, it is even more meaningful in the context of cancer drugs where the clinic purchases cancer drugs on behalf of CMS and is then reimbursed for them by both Medicare (80\%) and the beneficia- ries (20\%). Medicare does not reimburse community cancer clinics for uncollectible beneficiary coinsurance as it does for hospitals.

The combined impact of the prompt-pay problem, 2-quarter lag in ASP, and patient bad debt results in the Medicare reimbursement rate of ASP plus $6 \%$ in reality being far lower, often closer to ASP or less. This has been a key driver of community cancer clinics closures, especially in rural areas, and of their merging into hospital systems.

\section{Payment and policy advantages based on site of service}

Another key driver of the shift from community clinics to hospital systems is the steady erosion of revenues in the clinic setting because of the significant changes in Medicare payment policies for cancer care services. In addition, the wide variation of reimbursement for the identical services in the clinic versus outpatient hospital settings compounds the problem. For example, under the 2013 Medicare payment rules, hospitals are paid 51\% more than community cancer clinics for a representative mix of chemotherapy administration services. This differential would increase to $99 \%$ if CMS's reimbursement proposals for 2014 are implemented, meaning that Medicare will pay twice as much for chemotherapy administration services in the hospital versus community clinic setting.

As another example, under the 2013 Medicare payment rules, clinic and outpatient hospital payments are approximately the same across a representative mix of radiation therapy services, but the proposed 2014 rules would create a significant site-of-service differential with payments to hospitals approximately 55\% higher than community oncology clinics and freestanding radiation therapy centers. These types of discrepancies in reimbursement throughout oncology and other specialties greatly advantage hospital outpatient departments and, in effect, subsidize and encourage inefficiency. Hopefully, policymakers will take heed of the Medicare Payment Advisory Commission's (MedPAC) recent recommendation to level the playing field for outpatient services, including oncology services.

In addition to these code- and service-specific payment differentials outlined by MedPAC, hospitals enjoy other advantages relative to government policies relating to Part B drugs that contribute to the migration of cancer care toward hospitals. About a third of hospitals in the United States purchase cancer drugs through the $340 \mathrm{~B}$ program at discounts of up to $50 \%$, typically more than at least $30 \%$ below the Medicare reimbursement rate of ASP plus 6\%. ${ }^{7}$ This means that for $340 \mathrm{~B}$ hospitals, the margin on Medicare drugs can be as high as $100 \%$, whereas for clinics the margin is in actuality zero to minus $2 \%$. This explains why cancer 
drug spending is increasing so rapidly in the hospital outpatient setting and why cancer care is moving in that direction.

\section{The federal budget sequester}

The most recent challenge to access to cancer drugs and the viability of community-based cancer care is the federal government budget sequestration policy, and in particular, the administration's decision to apply this cut to both the 6\% services fee and also the acquisition cost of the underlying drugs purchased on behalf of CMS. The practical effect of the sequester cut is to effectively force cancer clinics to subsidize Medicare even more - that is, to make up the difference between what Medicare pays and the actual cost of cancer drugs.

Health care providers are never comfortable talking about their work in purely economic terms, but the fact remains that community cancer clinics are small businesses held to the economic reality that operating at a loss cannot be sustained. It is hard to imagine any business - small or otherwise - accepting a policy that requires operating at a loss. Oncologists should not be put in the untenable position of continuing to treat patients at a loss, which will result in clinic closings, or sending seniors to hospitals for treatment to keep the clinic doors open.

\section{Fixing the cancer care crisis for future generations}

The National Cancer Institute estimated that there were about 13.7 million Americans living with cancer in the United States last year. About 8 million of those are over the age of 65 years and about half of all cancer spending is associated with Medicare beneficiaries. ${ }^{8}$ As the baby boomers continue to reach 65 those numbers will only increase. So now is the time for policymakers to act to preserve cancer care access and stop the site-of-service shift into more costly hospital systems At a time when access and cost issues are intertwined, it is vital that payment amounts be commensurate with actual services provided, not the site of care. Preferentially paying higher amounts in certain settings will predictably lead to the expansion of higher-cost centers. The result will be further increases in the cost of cancer care for those who pay for it - patients, and private and government payers.

By circumstance and design, community oncology clinics have evolved over the past 2 decades into highly efficient clinical machines that treat, nurture, and restore America's cancer patients. Medicare should be subsidizing and taking action to maintain this modern miracle in high-quality outpatient cancer care instead of placing countless bureaucratic, legislative, and reimbursement burdens on its back.
Fortunately, legislation has been introduced in Congress to fix problems harming the nation's cancer care delivery. Bills in the House (HR 800) and Senate (S 806) would correct the prompt pay problem that artificially reduces Medicare drug reimbursement. Another bill in the House (HR 1416) would stop the sequester cut to the underlying cost of cancer drugs, and yet another House bill (HR 2869) would establish equal Medicare payment for the same services, regardless of whether the services are delivered in the clinic or outpatient hospital setting.

Over the next few months, many decisions about the future of our country and our health care delivery system will be made in Washington. Now is the time for the Congress to act to preserve modern community cancer care. And now it is time for physicians, nurses, allied professionals, and cancer patients to stand up to demand a viable and sustainable community oncology. We hope you will stand with us to drive change.

\section{References}

1. The Moran Company. Analyses for chemotherapy administration utilization and chemotherapy drug utilization, 2005-2011 for Medicare fee-for-service beneficiaries. https://media.gractions.com/ E5820F8C11F80915AE699A1BD4FA0948B6285786/01655fe97f3d-4d9a-80d0-d2f9581673a1.pdf. Released May 29, 2013. Accessed October 31, 2013

2. Community Oncology Alliance. Community oncology practice impact report: the changing landscape of cancer care. http:// www.communityoncology.org/UserFiles/Community_Oncology_ Practice_Impact_Report_6-25-13F.pdf. Issued June 25, 2013. Accessed October 31, 2013.

3. The Moran Company. Cost differences in cancer care across settings. https://media.gractions.com/E5820F8C11F80915AE699A1BD4 FA0948B6285786/adebd67d-dcb6-46e0-afc3-7f410de24657.pdf. Released August 2013. Accessed October 31, 2013.

4. Milliman Inc. Site of service cost differences for Medicare patients receiving chemotherapy. http://publications.milliman.com/ publications/health-published/pdfs/site-of-service-costdifferences.pdf. Released October 19, 2011. Accessed October 31, 2013.

5. Avalere Health LLC. Total cost of cancer care by site of service: physician office vs. outpatient hospital. http://www.communityoncology.org/pdfs/ avalere-cost-of-cancer-care-study.pdf. Released March 2012. Accessed October 31, 2013.

6. Milliman Inc. Comparing episode of cancer care costs in different settings: an actuarial analysis of patients receiving chemotherapy. http://us.milliman.com/insight/2013/Comparing-episode-ofcancer-care-costs-in-different-settings-an-actuarial-analysis-ofpatients-receiving-chemotherapy/. Released August 29, 2013. Accessed October 31, 2013.

7. US Department of Health and Human Services. OIG Memorandum Report: Payment for drugs under the hospital outpatient prospective payment system OEI-03-09-00420. http://oig.hhs.gov/oei/ reports/oei-03-09-00420.pdf. October 22, 2010. Accessed October 31, 2013.

8. Mariotto AB, Yabroff KR, Shao Y, Feuer EJ, Brown ML. Projections of the cost of cancer care in the United States: 2010-2020. J Natl Cancer Inst. 2011;103:117-128. 